\title{
Curvature Correction of the Hamilton-Jacobi Skeleton
}

\author{
Andrea Torsello and Edwin R. Hancock \\ Department of Computer science,University of York \\ York, YO10 5DD, UK \\ atorsell@cs.york.ac.uk
}

\begin{abstract}
The Hamilton-Jacobi approach has proved to be a powerful and elegant method for extracting the skeleton of a shape. The approach is based on the fact that the inward evolving boundary flux is conservative everywhere except at skeletal points. Nonetheless this method appears to overlook the fact that the linear density of the evolving boundary front is not constant where the front is curved. In this paper we present an analysis which takes into account variations of density due to boundary curvature. This yields a skeletonization algorithm that is both better localized and less susceptible to boundary noise than the Hamilton-Jacobi method.
\end{abstract}

\section{Introduction}

The skeletal abstraction of 2D and 3D objects has proved to be an alluring yet highly elusive goal for over 30 years in shape analysis. The topic is not only important in image analysis, where it has stimulated a number of important developments including the medial axis transform and iterative morphological thinning operators, but is also an important field of investigation in differential geometry and biometrics where it has lead to the study of the so-called morphological skeleton [4].

Given the importance of skeletal representations, the quest for reliable and efficient ways of computing skeletal shape descriptors has been a topic of sustained activity. The problem is a complex and elusive one because it is based on the detection of singularities on the evolution of the boundary. The available methods for extracting the skeleton can be divided into three broad categories. The first class of methods are those that involve the use of marching front techniques which simulate the grassfire transform. These methods are concerned with iteratively propagating the boundary front over time. Singularities in the simulated evolution of the front indicate the locations of the skeleton. This class of algorithms can be further divided into a) thinning methods [1, 2], b) methods where layers of pixels are sequentially pealed from the shape like the skin of an onion, and c) curve evolution methods [9, 23], where curve descriptors such as splines or snakes are transformed according to the eikonal equation. Thinning algorithms have a clear advantage in terms of simplicity. However, their performance is not invariant under Euclidean transformation. Curve evolution methods, on the other hand, are invariant under Euclidean transformation, but are mathematically less tractable. All three classes of marching front method encounter problems of numerical instability.

A second class of skeleton extraction algorithm is those that rely on the relationship between the Voronoi triangulation and the skeleton $[16,12,13]$. This work is based on the property that as the number of control points on the object boundary increases, then so the locus of the centers of the triangles of the corresponding Voronoi triangulation of the shape converge to the skeleton. The consequence is that as the triangulation increasingly approximates the shape boundary, then, correspondingly, the centers of the triangles increasingly approximate the skeleton. The important advantages of this approach are that it offers invariance under Euclidean transformation, that it is numerically stable, and it is of simple implementation. However, its major drawback is the relatively slow convergence speed of the skeleton approximation with respect to the number of control points on the boundary.

The third, and final, class of algorithms rely on the analysis of the differential structure of the boundary. An important method that falls into this class is that which results from the analysis of the boundary evolution dynamics using the Hamilton-Jacobi equations from classical mechanics [14]. This analysis leads to an eikonal equation which governs the boundary flow. Wherever this flow is nonsingular, the system is Hamiltonian, and, thus, conservative. However, when the system ceases to be conservative there are singularities in the flow of boundary evolution. When the boundary reaches the singularities a so-called shock forms. In the Hamilton-Jacobi setting skeletal points are detected by searching for points where the system ceases to be Hamiltonian. Analysis reveals that these are points where the divergence of the flow is non-zero $[6,19]$. The resulting skeleton search method is both algorithmically simple and 
numerically stable.

In the original formulation of the Hamilton-Jacobi method [19] there is a problem. The approach appears to overlook the fact that the linear density of the evolving boundary front is not constant where the front is curved. The result of changes in density is that the flux is not conservative and hence the premise underpinning the skeletonization method does not hold. Recently, Siddiqi et al. published a revised theory, rectifying the theoretical, if not the practical, shortcomings of the approach [20].

Hence, our aim in this paper is to perform a HamiltonJacobi analysis of boundary evolution under conditions where the density varies due to curvature. Instead of using the gradient of the distance map, i.e. the velocity field of the eikonal equation, we use the momentum field. In other words, we multiply the velocity by the linear density of the boundary-front. The resulting field is conservative. This leads to a new skeleton extraction method. We compare the curvature corrected skeletonisation method with the original Hamilton-Jacobi method. The advantages of the new method are improved localization and stability, espectially of the endpoints of the skeletal branches.

\section{Hamilton-Jacobi Skeleton}

We commence by defining a distance-map that assigns to each point on the interior of an object the closest distance $D$ from the point to the boundary (i.e. the distance to the closest point on the object boundary). The gradient of this distance-map is a field $\vec{F}$ whose domain is the interior of the shape. The field is defined to be $\vec{F}=\nabla D$, where $\nabla=\left(\frac{\partial}{\partial x}, \frac{\partial}{\partial y}\right)^{T}$ is the gradient operator. The trajectory followed by each boundary point under the eikonal equation is governed by the ordinary differential equation $\dot{\vec{x}}=\vec{F}(\vec{x})$, where $\vec{x}$ is the coordinate vector of the point. Siddiqi, Bouix, Tannenbaum, and Zucker assume that this dynamic system is Hamiltonian everywhere except on the skeleton. This assumption implies that at skeletal points the field $\vec{F}$ is conservative, i.e. $\nabla \cdot \vec{F}=0$. However, the total inward flux through the boundary of the shape is non zero. In fact, the flux is proportional to the length of the boundary.

The divergence theorem states that the integral of the divergence of a vector-field over an area is equal to the flux of the field over the enclosing boundary of that area. In our case, this implies that

$$
\int_{A} \nabla \cdot \vec{F} d \sigma=\int_{L} \vec{F} \cdot \vec{n} d l=\Phi_{A}(\vec{F}),
$$

where $A$ is an arbitrary area, $\vec{F}$ is a vector field defined in $A$, $d \sigma$ is the area differential in $A, d l$ is the length differential on the border $L$ of $A$, and $\Phi_{A}(\vec{F})$ is the outward flux of $F$ through the border $L$ of the area $A$.
By virtue of the divergence theorem within the interior of the shape, there are points where the system is not conservative. The non-conservative points are those where the boundary trajectory is not well defined, i.e. where there are singularities in the evolution of the boundary. These points are the so-called shocks or skeleton of the shape- boundary. Shocks are thus characterized by locations where $\nabla \cdot \vec{F}<0$.

\section{Curvature in the Boundary Front}

Unfortunately, the hypothesis that the field $\vec{F}$ is conservative does not hold in general. To illustrate this point, let us consider an instant $t$ in the inward boundary evolution. The initial shape boundary has evolved under the eikonal equation to the front $S^{t}$ which is at every location orthogonal to $\vec{F}$. We would like to select a point $p \in S^{t}$ and compute the value of $\nabla \cdot \vec{F}(p)$ at this point. Since the divergence operator is invariant under rotations, we can write:

$$
\nabla \cdot \vec{F}=\frac{\partial}{\partial v_{\|}} F+\frac{\partial}{\partial v_{\perp}} F
$$

where $v_{\|}=\vec{F}(p)$ and $v_{\perp}$ is a normal vector orthogonal to $v_{\|}$. Moreover, since $\|F\|=1$ everywhere, then $\frac{\partial}{\partial v_{\|}} F=0$. However, it is also the case that $\frac{\partial}{\partial v_{\perp}} F(p)=-\kappa(p)$ where $\kappa(p)$ is the curvature in $p$ of the border front $S^{t}$ oriented so that $\kappa(p)$ is positive if the osculating circle is in the interior of the front. Hence, we have $\nabla \cdot \vec{F}(p)=-\kappa(p)$, In other words, the divergence $\nabla \cdot \vec{F}$ is not always zero as predicted by the Hamilton-Jacobi approach. Rather, it is equal to the curvature of the front of the inward evolving boundary.

As a concrete example, consider a circle of unit radius centered in $(0,0)^{T}$. the gradient of the distance map at point $(x, y)^{T}$ is $\nabla D=-\frac{1}{\sqrt{x^{2}+y^{2}}}(x, y)^{T}$, and the divergence is $\nabla^{2} D=-\frac{1}{\sqrt{x^{2}+y^{2}}} \neq 0$.

The fact that the divergence is non-zero can be easily understood by appealing to an analogy from physics. Let us assume that a fluid of uniform density flows from the border of the shape, which acts as a source, to the skeleton, which acts as a sink. If the fluid is incompressible, then the fluid density never changes and the velocity field $\vec{F}$ is conservative everywhere except at points on the boundary skeleton. If, on the other hand, the fluid is compressible, then as soon as a curved front compresses the fluid, the density changes and the velocity field is no longer conservative.

To develop this idea one step further, consider a segment $d l(t)$ of the boundary front $S^{t}$ at time $t$. We assume that this segment has average linear density $\hat{\rho}(t)$ (see Figure 1). Under the eikonal equation, at time $t+\Delta t$ the boundary front segment $d l(t)$ has evolved to $d l(t+\Delta t)$. Since each of the points in $d l(t)$ are now contained in $d l(t+\Delta t)$, the total 


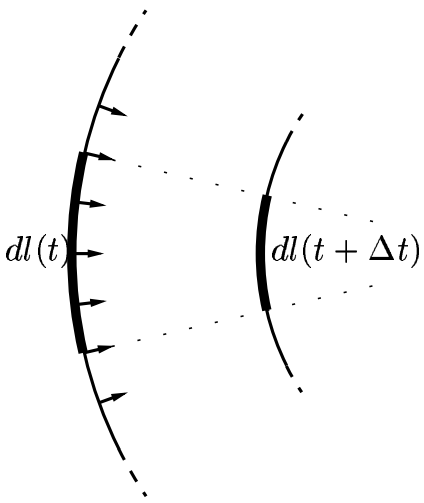

Figure 1: Evolution of a boundary segment.

mass of the two segments is the same. However, if $d l(t)$ is curved then the lengths of the segments are different: ||$l(t+$ $\Delta t)\|\neq\| l(t) \|$. Thus the average density in $l(t+\Delta t)$ is $\hat{\rho}(t+\Delta t) \neq \hat{\rho}(t)$. As a result, when the front is curved, then the density is not constant and we have to take into account mass effects. That is, we have to resort to the more general principle of conservation of momentum.

\section{Momentum field}

Based on this physical intuition we state that there is indeed a conservative field associated with the dynamics of the boundary, namely the momentum $\vec{M}=\rho \vec{F}$, where $\rho$ is the scalar field that assigns to each point the linear density of the boundary front. As a result we have that

$$
\nabla \cdot(\rho \vec{F})=0,
$$

Applying the rules of product differentiation, we obtain the partial differential equation (PDE):

$$
\nabla \rho \cdot \vec{F}=-\rho \nabla \cdot \vec{F} .
$$

By setting $\sigma=\log (\rho)$, we can write the above PDE as a function of the log-density $\sigma$ :

$$
\nabla \sigma \cdot \vec{F}=-\nabla \cdot \vec{F} .
$$

This is a transport equation that can be reduced to a set of ordinary differential equations (ODE) along the paths of the boundary points.

$$
\left\{\begin{array}{l}
\frac{d}{d t} \sigma(s(t))=-\nabla \cdot \vec{F}(s(t)) \\
\frac{d}{d t} s(t)=\vec{F}(s(t))
\end{array}\right.
$$

We can derive this equation by analyzing the change of density of the segment $d l$ in Figure 1. We know that $\hat{\rho}(t)\|d l(t)\|=m$. where $d l(t)$ is the evolution of the border segment $d l$ at time $t, m$ is its mass, and $\hat{\rho}(t)$ and $\kappa(t)$ are the segment's average linear density and curvature at time $t$. After a small interval of time $\Delta t$, the segment length will be

$$
\|d l(t+\Delta t)\|=\|d l(t)\| \frac{\kappa(t)}{\kappa(t+\Delta t)}+O\left(\Delta t^{2}\right),
$$

and the curvature

$$
\kappa(t+\Delta t)=\frac{\kappa(t)}{1-\kappa(t) \Delta t}+O\left(\Delta t^{2}\right) .
$$

From these equations and the conservation of mass, we have:

$\hat{\rho}(t+\Delta t)=\frac{m}{\|d l(t+\Delta t)\|}=\hat{\rho}(t) \frac{1}{1-\kappa(t) \Delta t}+O\left(\Delta t^{2}\right)$.

Hence

$$
\hat{\rho}(t+\Delta t)-\hat{\rho}(t)=\hat{\rho}(t) \frac{\kappa(t) \Delta t}{1-\kappa(t) \Delta t}+O\left(\Delta t^{2}\right) .
$$

Taking the limit for $\Delta t \rightarrow 0$ and $\|d l\| \rightarrow 0$, we have:

$$
\frac{\frac{d}{d t} \rho(s(t))}{\rho(s(t))}=\kappa(s(t))
$$

where $s(t)$ is the trajectory to which the limit point of the segment $d l$ tends as $\|d l\| \rightarrow 0$ under the eikonal equation. Integrating (4) we obtain:

$$
\log (\rho(s(t)))=\int_{0}^{t} \kappa(s(\tau)) d \tau=-\int_{0}^{t} \nabla \cdot \vec{F}(s(\tau)) d \tau .
$$

\section{Computing the density}

To obtain the momentum field we need to integrate the density field over the interior of the shape. Since images have a finite resolution, we need to discretize the solution onto the image lattice.

One approach is to express the PDE (2) as a system of difference equations. The difference equations form a linear system that can then be solved to obtain the $\log$-density $\sigma=$ $\log (\rho)$. The problem with this approach is that the skeleton is a set of singularities of the momentum field. Hence the density can have very different values at opposite sides of a skeletal branch. The result is that the linear system will have no solution. Infact, even seaking an approximate a solution using a residual descent method would result in oscillations near the skeleton. 


\subsection{Integration in Time}

In order to overcome this problem we need to ensure that the difference operator used in the equations never crosses a skeletal branch. One way to guarantee this is to integrate the equation in the time domain. This must be done so that the formulae giving the value of $\rho$ at points on the boundary front at time $t$ reference values of $\rho$ only at points in the fronts at previous times. We can realize this by integrating the ODE (3) along the paths of the boundary points.

To do this we opt to use the second order CranckNicolson method [7]. For each point $(x, y)$ in the interior of the shape, we have the equation:

$\sigma(s(t))=\sigma(s(t-1))-\frac{1}{2}[\nabla \cdot \vec{F}(s(t))+\nabla \cdot \vec{F}(s(t-1))]$.

Using this equation we can calculate the log-density at a point on the evolving boundary at time $t$, referencing only values of the log-density at points that belong the front at previous times. Since the evolution never crosses the skeleton, we are guaranteed not to cross skeletal branches during our calculations.

\subsection{Integration in Space}

Equation (5) allows us to integrate the $\log$-density $\sigma$ in the time domain along the path $s$. However, we have not shown how to calculate the integration path. Fortunately, we do not need to calculate every possible path. Let us assume that at time $t$ the boundary front passes through the point $s(t)=(x, y)^{T}$. The first order approximation of the position of this point at time $t-1$ is $s(t-1)=$ $(x, y)^{T}-\vec{F}(x, y)=\left(x-F_{x}, y-F_{y}\right)^{T}$. Using this approximation, we can write Equation (5) in the spatial domain instead of the time domain:

$$
\begin{aligned}
& \sigma(x, y)=\sigma\left(x-F_{x}(x, y), y-F_{y}(x, y)\right) \\
& \left.-\frac{1}{2}[\nabla \cdot \vec{F}(x, y))+\nabla \cdot \vec{F}\left(x-F_{x}(x, y), y-F_{y}(x, y)\right)\right] .
\end{aligned}
$$

As shown in Figure 2, the point $(x, y)^{T}-\vec{F}(x, y)$ does not belong to the image lattice. Hence we need to interpolate it using the values at the four corners of the square containing the point. We opt to compute the quantity $f(x+a, y+b)$ with $a, b \in[0,1)$ using the formula

$$
\begin{aligned}
& (a-1)(b-1) f(x, y)+a(b-1) f(x+1, y) \\
& \quad+(a-1) b f(x, y+1)+a b f(x+1, y+1) .
\end{aligned}
$$

With this interpolation Equation (5) becomes:

$$
\begin{aligned}
& {\left[1-\left(1-\left|F_{x}\right|\right)\left(1-\left|F_{y}\right|\right)\right] \sigma(x, y)=\left|F_{x}\right|\left(1-\left|F_{y}\right|\right) \sigma\left(x^{\prime}, y\right)} \\
& \quad+\left(1-\left|F_{x}\right|\right)\left|F_{y}\right| \sigma\left(x, y^{\prime}\right)+\left|F_{x}\right|\left|F_{x}\right| \sigma\left(x^{\prime}, y^{\prime}\right) \\
& \left.\quad-\frac{1}{2}[\nabla \cdot \vec{F}(x, y))+\nabla \cdot \vec{F}\left(x-F_{x}, y-F_{y}\right)\right], \quad \text { (6) }
\end{aligned}
$$

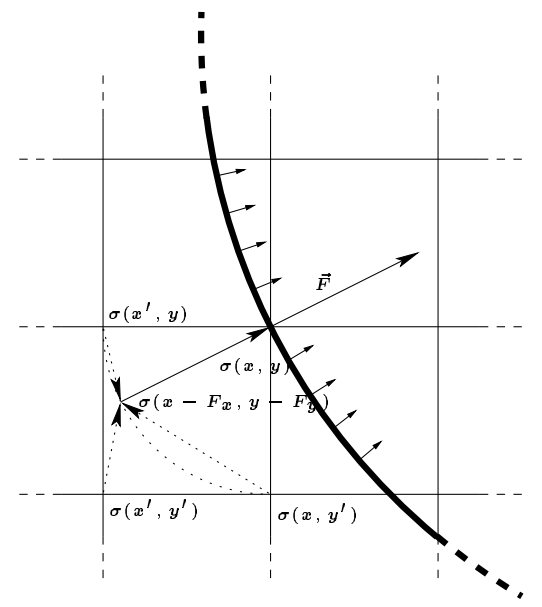

Figure 2: Integration along the boundary path.

where $x^{\prime}=x+\operatorname{sgn}\left(F_{x}(x, y)\right)$ and $y^{\prime}=y+\operatorname{sgn}\left(F_{y}(x, y)\right)$.

Using Equation (6) we can compute the value of the $\log$-density $\sigma(x, y)$ using values of $\sigma$ at the points spanned by the evolving boundary front before the point $(x, y)^{T}$. Hence, to calculate $\sigma$ all we need do is to iterate Equation (6) through the interior points according to front arrival time. We start from the points reached first by the boundary front and proceed to those reached last. Since the evolving boundary front is moving with constant unit velocity, the time taken by the front to reach the point with position $(x, y)^{T}$ is equal to its distance from the initial shape boundary.

\section{Skeletonization}

Once the divergence of the momentum field is to hand, we can extract the skeleton. The extraction process is performed by thinning the shape by removing border points that have energy absorption below a certain threshold, and whose removal would not cause the shape to be split into two disjoint parts. The remaining shape is further thinned to a 1-pixel wide skeleton, paying attention to maintaining the connectivity of the shape and to avoiding shortening of the skeleton by eliminating endpoints. Expressed in terms of pseudo-code the thinning process of the shape $S$ is as follows:

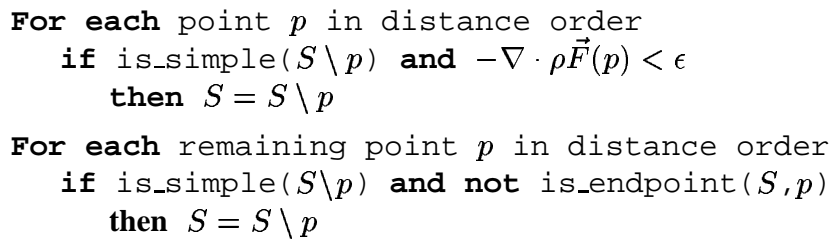

The predicate is_simple determines whether the shape 
is still connected after the removal of the point $p$. It does so by checking only the points in the neighborhood of $p$. The shape $S \backslash p$ is connected if the points in the neighborhood of $p$, excluding $p$, are connected. Similarilly, is_endpoint determines whether $p$ is an endpoint. It does so only by inspecting the neighborhood of $p$. The point is an endpoint if it has at most two neighboring points, and these points are horizontally or vertically adjacent.

\section{Experimental Comparison}

In this section we attempt to characterize the differences between the Hamilton-Jacobi skeletonization method and our density-corrected approach. We start by providing a qualitative analysis of the difference in the divergence of the velocity and momentum fields. Secondly, we provide an analysis of the noise and thresholding sensitivity of the two methods. Finally we provide a more quantitative analysis of the localization properties of the two skeletonization methods.

Figure 3 shows, for a few selected shapes from our database, the values of the divergence of the velocity field $\nabla$. $\vec{F}, \log (\rho)$, and $\nabla \cdot \vec{\rho} F$. In these pictures white (grey-scale 255 ) corresponds to a large positive value, black (grey scale value 0 ) to a large negative value and zero is represented by the grey scale value 128 .

It is clear from the pictures that the divergence of the velocity field is not zero in correspondence with a curved boundary. Furthermore, quantization in the localization of the shape causes the initial boundary to be very jagged. This high-frequency, low-amplitude noise is transported and amplified throughout the velocity field. This in turn yields a noisy and poorly localized skeleton. By contrast, the density correction in the momentum field dampens the noise.

\subsection{Noise Sensitivity}

To counter quantization noise from the object boundary, we need to smooth the distance map and select an appropriate skeletonization threshold. If either the smoothing radius or the threshold is too large, then some of the branches of the skeleton will be thinned away. If, on the other hand, the selected values are too small, then the detected skeleton will have large numbers of spurious branches (See Figure 4). In this section we characterize the effects of the smoothing radius and the skeletonization threshold on the quality of the detected skeleton.

Figure 4 displays the effects of very low (top) and very high (bottom) values of the smoothing radius and the skeletonization threshold on a test shape. The picture shows, left to right, the divergence of the velocity field, the uncorrected Hamilton-Jacobi skeleton, the divergence of the momentum field, and the skeleton extracted using the density-
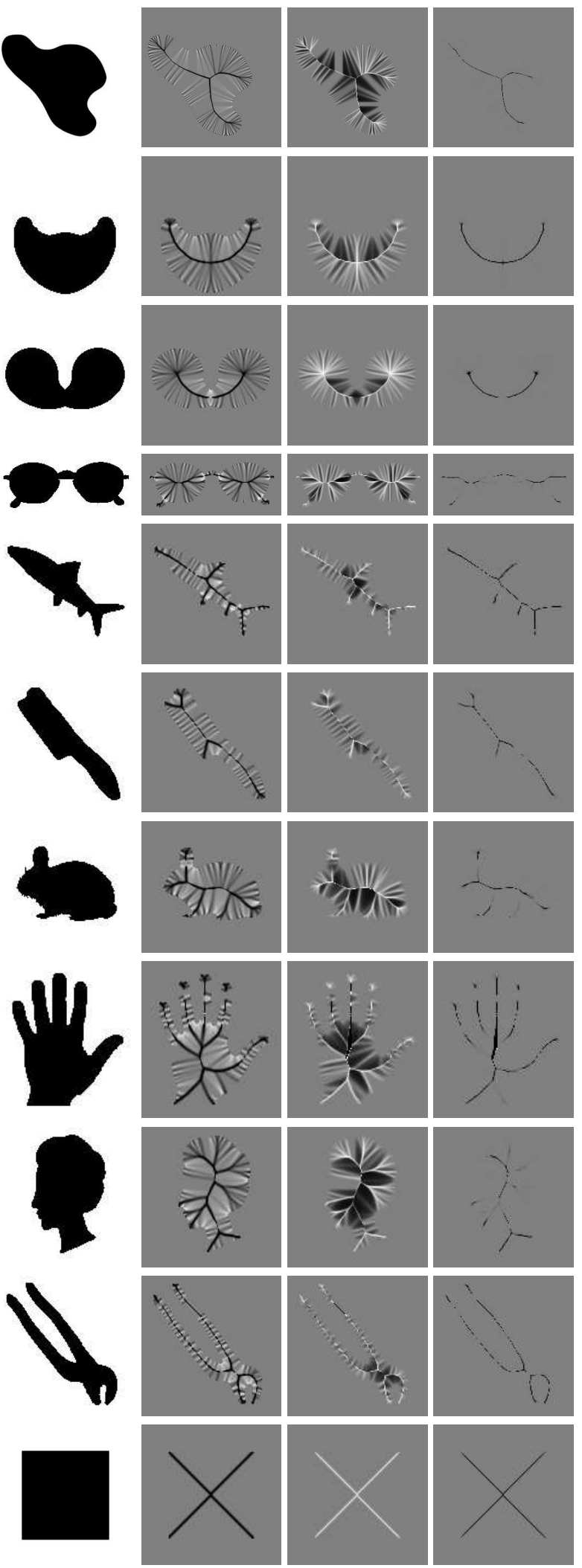

Figure 3: Differences in the velocity and momentum fields. Left to right: shape, $\nabla \cdot \vec{F}, \log (\rho)$, and $\nabla \cdot \vec{\rho} F$ 

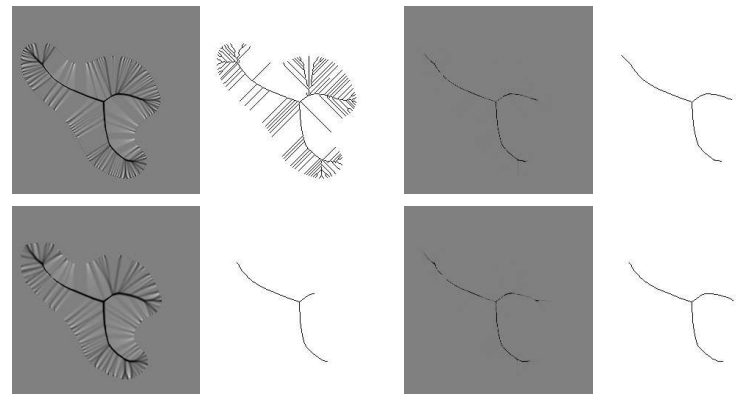

Figure 4: The effect of smoothing on skeleton extraction.

corrected method. These pictures demonstrate that the density corrected method is much less sensitive to the amount of smoothing and to the value of the threshold.

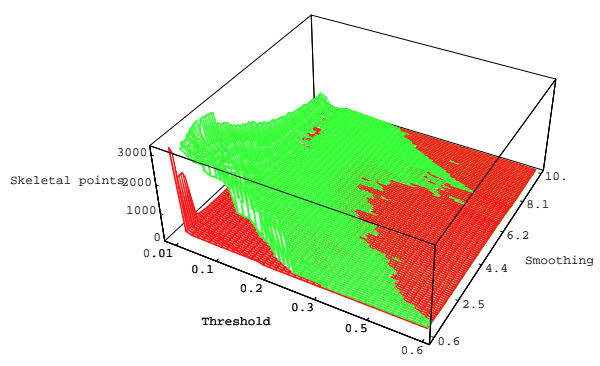

(a) Both

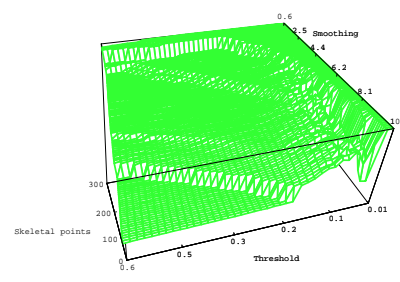

(b) Hamilton-Jacobi

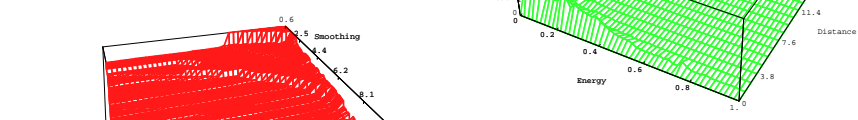

(a) Hamilton-Jacobi

faster as we increase the threshold or the smoothing radius. Moreover, it maintains the plateau for longer. Figures $5 \mathrm{~b}$ and $5 \mathrm{c}$ show the results separately for the Hamilton-Jacobi and the density-corrected methods. Here location closest to the viewer direction is the plateau side of the plots. The ridges in the forefront show the drop in the number of skeletal points due to the smoothing away of an image feature. In both cases, the drop is sudden but the ridge in the HamiltonJacobi plot shows a higher dependence on the threshold.

\subsection{Skeleton Localization}

In this section we characterize the localization properties of the skeleton extracted using the Hamilton-Jacobi method and the new density corrected method on a wide variety of shapes. To this end, we investigate how the values of the divergence of the velocity and of the momentum field are distributed over the distance to the extracted skeleton. Figure 6 plots a histogram of the distribution of non-skeletal points as a function of distance and divergence value for the test shapes. The figure shows that the Hamilton-Jacobi skeleton has a non-negligible tail for high divergence values, even at large distance from the extracted skeleton.

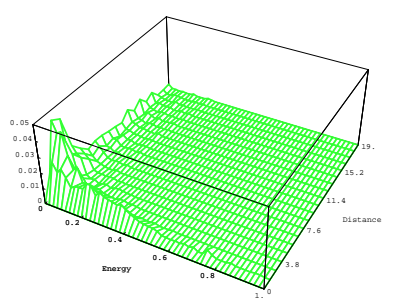

Figure 6: Histogram over val the field and distance to skeleton

We have also performed an experiment aimed at quantifying the localization of the skeleton on a database of shapes. We have used a database of 50 shapes and have histogrammed the distribution of field divergence as a function of the distance to the skeleton. We have repeated this procedure for both the velocity field and the momentum field. For each shape, we take the mean of the relevant divergencedistribution as a measure of divergence-localization.

Figure 7 shows histograms of this divergencelocalization measure over all the shapes in our database. In Figure $7 \mathrm{a}$ we show the localization histogram for the velocity field. The mean of this distribution is 2.52 , while the variance is 0.34 . Figure $7 \mathrm{~b}$ is the corresponding histogram for the momentum field. The mean of this distribution is 1.46 , while the variance is 0.28 . The density correction clearly leads to a better localization of the skeleton. 


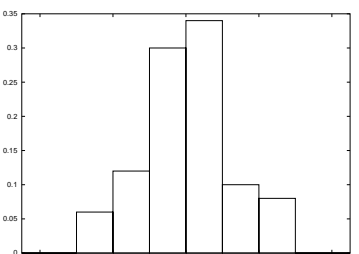

(a) Hamilton-Jacobi

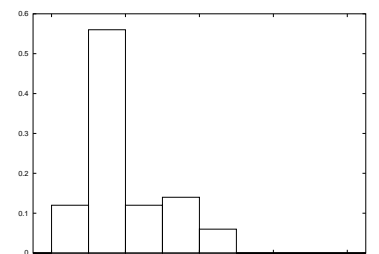

(b) Density corrected
Figure 7: Histogram of divergence-localization on a database of 50 shapes.

\section{Conclusions}

In this paper we have provided a proof that a key hypothesis underpinning the Hamilton-Jacobi framework, namely that the velocity field is conservative, does not hold in the presence of curved boundaries. This is due to the increase of point density for curved front boundary evolution. To overcome this problem, we have presented an analysis which takes into account variations of density due to boundary curvature. This yields a skeletonization algorithm that is both better localized and less susceptible to boundary noise than the Hamilton-Jacobi method.

\section{References}

[1] C. Arcelli and G. Sanniti di Baja. A width-independent fast thinning algorithm. IEEE TPAMI, 7(4):463-474, 1985.

[2] C. Arcelli and G. Sanniti di Baja. Ridge points in euclidean distance maps. Pattern Rec. Letters, 13:237-243, 1992.

[3] J. August, A. Tannenbaum, and S. W. Zucker. On the evolution of the skeleton. In ICCV, pp. 315-522, 1999.

[4] H. Blum. Biological shape and visual science (part i). Journal of theoretical Biology, 38:205-287, 1973.

[5] G. Borgefors, G. Ramella, and G. Sanniti di Baja. Multiscale skeletons via permanence ranking. In Advances in $\mathrm{Vi}$ sual Form Analysis, pages 31-42. World Scientifi c, 1997.

[6] S. Bouix and Kaleem Siddiqi. Divergence-based medial surfaces. In Computer Vision ECCV 2000, volume 1 of LNCS, pages 603-618. Springer, 2000. LNCS 1842.

[7] J. Crank and P.Nicolson, A practical method for numerical evaluation of solutions of partial differential equations of the heat conduction type. In Proc. Cambridge Philos. Soc., Vol. 43, pp. 50-67, 1947.

[8] P. Klein, S. Tirthapura, D. Sharvit, and B. B. Kimia. A tree-edit-distance algorithm for comparing simple, closed shapes. In ACM-SIAM Symp. on Discrete Algorithms, 1999.

[9] F. Leymarie and M. D. Levine. Simulating the grassfi re transform using an active contour model. IEEE TPAMI, 14(1):56-75, 1992.
[10] B. Luo et al. A probabilistic framework for graph clustering. In $C V P R$, volume I, pages 912-919. IEEE Computer Society, 2001.

[11] D. Macrini et al. View-Based 3-D Object Recognition using Shock Graphs. In ICPR, pages 24-28. IEEE Computer Society, 2002.

[12] R. L. Ogniewicz. A multiscale mat from voronoi diagrams: the skeleton-space and its application to shape description and decomposition. In Aspects of Visual Form Processing, pages 430-439. World Scientifi c, 1994.

[13] R. L. Ogniewicz and O. Kübler. Hierarchic voronoi skeletons. Pattern Recognition, 28(3):343-359, 1995.

[14] S. J. Osher and J. A. Sethian. Fronts propagating with curvature dependent speed: Algorithms based on hamilton-jacobi formulations. J. of Computational Physics, 79:12-49, 1988.

[15] M. Pelillo, K. Siddiqi, and S. W. Zucker. Matching hierarchical structures using association graphs. IEEE TPAMI, 21(11):1105-1120, 1999.

[16] M. Schmitt. Some examples of algorithms analysis in computational geometry by means of mathematical morphological techniques. In Geom. and Robotics, 1989. LNCS 391.

[17] T. S. Sebastian, P. N. Klein, and B. B. Kimia. Recognition of shapes by editing shock graphs. In ICCV, volume I, pages 755-762, 2001.

[18] A. Shokoufandeh, S. J. Dickinson, K. Siddiqi, and S. W. Zucker. Indexing using a spectral encoding of topological structure. In $C V P R, 1999$.

[19] K. Siddiqi, S. Bouix, A. Tannenbaum, and S. W. Zucker. The hamilton-jacobi skeleton. In ICCV, pages 828-834, 1999.

[20] K. Siddiqi, S. Bouix, A. Tannenbaum, and S. W. Zucker. Hamilton-Jacobi skeletons. Int. J. of Computer Vision, 3:215-231, 2002.

[21] K. Siddiqi and B. B. Kimia. A shock grammar for recognition. In CVPR, pages 507-513, 1996.

[22] K. Siddiqi, A. Shokoufandeh, S. J. Dickinson, and S. W. Zucker. Shock graphs and shape matching. Int. J. of Computer Vision, 35(1):13-32, 1999.

[23] H. Tek and B. B. Kimia. Symmetry maps of free-form curve segments via wave propagation. In ICCV, pages 362-369, 1999.

[24] S. Tirthapura, D. Sharvit, P. Klein, and B. B. Kimia. Indexing based on edit-distance matching of shape graphs. In SPIE International Symposium on Voice, Video, and Data Communications, pages 25-36, 1998.

[25] A. Torsello and E. R. Hancock. Effi ciently computing weighted tree edit distance using relaxation labeling. In EMMCVPR, pages 438-453, 2001. LNCS 2134. 